\title{
Coronavirus Disease Clinical and Laboratory Parameters: Dismembering the Values Reveals Outcomes
}

Tehzeeb Zehra ${ }^{1}$, Shahzad K. Siddique ${ }^{2}$, Rahila Aamir ${ }^{1}$, Adil Mahmood ${ }^{2}$, Abdul Hameed Kiani ${ }^{2}$, Sana T. Virk ${ }^{3}$

1. Internal Medicine, Shifa Tameer-E-Millat University, Shifa College of Medicine, Islamabad, PAK 2. Internal Medicine, Shifa International Hospital, Islamabad, PAK 3. Internal Medicine, Air University Islamabad, Islamabad, PAK

Corresponding author: Tehzeeb Zehra, drteh.ali@gmail.com

\section{Abstract}

\section{Background}

The medical community's understanding of the novel coronavirus disease (COVID-19) was limited initially, and many laboratory investigations were performed to observe effects of the virus on the body, its complications, and outcomes. We observed that some laboratory investigations provided redundant information regarding outcomes, and, therefore, were not necessary. Therefore, the extent of laboratory investigations may need to be pared down to not only avoid issues related to repeated blood sampling but also to minimize the financial burdens in poor socioeconomic countries.

\section{Objective}

This study aimed to observe trends of clinical and laboratory values in COVID-19 patients and their relationship to outcomes, including disease severity, length of hospital stay, and mortality.

\section{Methods}

We conducted an observational cohort study of COVID-19 patients treated as inpatients at the Shifa International Hospital (SIH) in Islamabad in April 2020. Patients were included if they were nonsurgical, adult inpatients of SIH diagnosed with COVID-19 via positive polymerase chain reaction test. We monitored study participants' clinical and laboratory values (including hypoxia) on admission and throughout the study period. We used IBM SPSS Statistics for Windows, Version 23.0 (IBM Corp., Armonk, NY, USA) for data entry and analysis. Descriptive statistics were calculated for qualitative and quantitative data. We determined the effect of all variables on outcomes through chi-squared or Fisher's exact test, and p-values $<0.05$ with $95 \%$ confidence interval were considered statistically significant.

\section{Results}

Review began 02/22/2021 Review ended 03/01/2021 Published 03/05/2021

\section{() Copyright 2021}

Zehra et al. This is an open access article distributed under the terms of the Creative Commons Attribution License CC-BY 4.0., which permits unrestricted use, distribution, and reproduction in any medium, provided the original author and source are credited.
A total of 51 patients with COVID-19 were enrolled. Most of the study participants were men older than age 50 with multiple comorbidities and resided in Khyber Pakhtunkhwa. Length of hospital stay ranged from eight to 14 days, and most patients had severe disease and survived. Factors such as patient age, gender, comorbid conditions, residence, and medication did not significantly affect outcomes. Hypotension during the height of symptoms and oxygen saturations $<80 \%$ on admission was associated with prolonged hospital stays. Two complete blood count (CBC) parameters (platelet counts and mean corpuscular volume, MCV) were strongly associated with mortality and severity in our patients. Four non-CBC parameters (alanine transaminase, ALT; D-dimer; C-reactive protein, CRP; and lactate dehydrogenase, LDH) had strong statistical impact on disease severity, length of hospital stay, and mortality in our patients.

\section{Conclusion}

In a resource-limited country, laboratory testing must be chosen wisely and used appropriately. Patient age, gender, comorbid conditions, drugs, residence, and ferritin levels did not affect COVID-19 outcomes. Hemoglobin, platelet count, MCV, CRP, D-dimer, ALT, LDH, hypoxia, and hypotension were all correlated to disease outcomes. Therefore, these factors are useful laboratory examinations for COVID-19 patients, especially in poor countries.

Categories: Internal Medicine, Infectious Disease

Keywords: covid-19, trends of laboratory values in covid-19, outcome predictors in covid-19, curtail laboratory testing in covid-19 patients, serial laboratory testing in covid -19

\section{Introduction}

The novel coronavirus disease (COVID-19) needs little introduction since December 2019, when the 
healthcare workers in the city of Wuhan, China, first discovered cases at the start of the COVID-19 epidemic [1]. As of November 1, 2020, nearly 46 million cases and 1.2 million deaths have been reported globally due to COVID-19, while the pandemic continues as a Public Health Emergency of International Concern, according to the World Health Organization (WHO) [2].

While various theories have been proposed regarding the initial onset and spread of COVID-19, research suggests that the virus originated from bats [3]. COVID-19 is a febrile illness with a broad spectrum of respiratory involvement and may progress to involve multiple systems. Leukopenia, elevated c-reactive protein (CRP) levels, thrombocytopenia, and acute kidney injury were common laboratory findings associated with the disease [4-6].

Many drugs have been explored as potential treatments for COVID-19 [7], and research is ongoing for vaccine trials and therapies, including immunoglobulin, convalescent plasma, antiviral agents, and other modalities.

The novelty of the severe acute respiratory syndrome coronavirus-2 (SARS-CoV-2) steered the overwhelming trend of laboratory investigation during the follow-up of COVID-19 patients. Reviewing SARS-CoV-2 research data from time to time, we observed that too many investigations are performed to predict the outcome of patients, contributing to a substantial financial and psychological burden on these patients. Therefore, we evaluated the association between laboratory investigations and their effects on COVID-19 outcomes to determine if multiple laboratory evaluations are clinically justified.

\section{Materials And Methods}

We conducted an observational cohort study of COVID-19 patients treated as inpatients at the Shifa International Hospital (SIH) in Islamabad in April 2020. The ethical review board of SIH approved the study design. The study included inpatients of SIH diagnosed with COVID-19 via positive polymerase chain reaction testing in April 2020. The study excluded all surgical patients, pediatric patients (i.e., those younger than 15 years), chemotherapy patients, and patients with a hospital stay shorter than 48 hours. We also excluded any patients who did not receive follow-up laboratory evaluations.

We recorded study variables from patients' medical records. Laboratory values on admission and their trends during worsening of oxygen saturation were recorded and entered in IBM SPSS Statistics for Windows, Version 23.0 (IBM Corp., Armonk, NY, USA). Patient data were compared to United States standard values for interpretation. Severity was graded according to WHO criteria for COVID-19 severity. We calculated descriptive statistics for both qualitative and quantitative data. The effect of all variables on outcomes was calculated using chi-squared or Fisher's exact tests, and p-values $<0.05$ with $95 \%$ confidence interval were considered statistically significant.

\section{Results}

A total of 51 patients with COVID-19 were enrolled. As shown in Table 1, most of our study patients were men older than age 50 with multiple comorbidities and resided in the Khyber Pakhtunkhwa district. A hospital stay of eight to 14 days was the most common, and most patients had severe disease. Only six patients $(11.8 \%)$ died, however. Age, gender, comorbid conditions, and residence had no significant effect on disease severity, length of hospital stay, or mortality. 


\section{Cureus}

\begin{tabular}{|c|c|c|}
\hline Age (years) & Number of cases & $\%$ \\
\hline$<30$ & 0 & 0 \\
\hline 30 to 50 & 15 & 29.4 \\
\hline 50 to 70 & 31 & 60.78 \\
\hline$>70$ & 5 & 9.8 \\
\hline Mean \pm SD & $57.2 \pm 10.28$ & \\
\hline \multicolumn{3}{|l|}{ Gender } \\
\hline Male & 41 & 80.4 \\
\hline Female & 10 & 19.6 \\
\hline \multicolumn{3}{|l|}{ Residence } \\
\hline KPK & 19 & 37.3 \\
\hline Punjab & 17 & 33.3 \\
\hline Islamabad & 14 & 27.5 \\
\hline Baluchistan & 1 & 2.0 \\
\hline \multicolumn{3}{|l|}{ Comorbidities } \\
\hline Diabetes mellitus & 9 & 17.6 \\
\hline Hypertension & 7 & 13.7 \\
\hline Ischemic heart disease & 1 & 2.0 \\
\hline Chronic kidney disease & 1 & 2.0 \\
\hline Malignancy & 2 & 3.9 \\
\hline Multiple & 12 & 37.3 \\
\hline Nil & 19 & 23.5 \\
\hline \multicolumn{3}{|l|}{ Severity } \\
\hline Mild & 12 & 23.5 \\
\hline Moderate & 12 & 23.5 \\
\hline Severe & 20 & 39.2 \\
\hline Critical & 7 & 13.7 \\
\hline Mortality & 6 & 11.8 \\
\hline \multicolumn{3}{|l|}{ Length of hospital stay } \\
\hline 1 to 7 days & 19 & 37.3 \\
\hline 8 to 14 days & 20 & 39.2 \\
\hline to 28 days & 12 & 23.5 \\
\hline
\end{tabular}

TABLE 1: Baseline characteristics of patients and frequencies of outcome $(n=51)$

KPK, Khyber Pakhtunkhwa; SD, standard deviation

During maximum symptoms, nine patients had blood pressure $<110 / 70 \mathrm{mmHg}$ and most of those patients (55.6\%) had extended hospital stays ( 14 to 28 days; $\mathrm{p}=0.055$; Table 2 ). $\mathrm{SpO}_{2}$ value on admission strongly affected the length of hospital stay. Among patients presenting with $<80 \%$ saturation, most of them had more extended hospital stays (14 to 28 days) than those with higher saturations $(\mathrm{p}=0.000)$. We did not correlate oxygen saturation levels with severity of disease in our patients because a patient's disease 


\section{Cureus}

severity was categorized mainly on the basis of oxygen saturation, so increasing level of hypoxia was associated with more severe illness by default.

\begin{tabular}{|c|c|c|c|}
\hline Variable & Length of hospital stay p-values & Severity p-values & Mortality p-values \\
\hline BP on admission & 0.34 & 0.47 & 0.53 \\
\hline BP during maximum symptoms & 0.055 & 0.28 & 0.55 \\
\hline Room air oxygen saturation on admission & 0.000 & NA & 0.48 \\
\hline TLC on admission & 0.27 & 0.15 & 0.15 \\
\hline TLC trend during worsening of symptoms & 0.12 & 0.023 & 0.53 \\
\hline Platelets on admission & 0.78 & 0.72 & 0.39 \\
\hline Platelets trend during worsening of symptoms & 0.073 & 0.016 & 0.044 \\
\hline MCV on admission & 0.96 & 0.58 & 0.43 \\
\hline MCV trend during worsening of symptoms & 0.27 & 0.41 & 0.049 \\
\hline Hemoglobin on admission & 0.73 & 0.05 & 0.75 \\
\hline Hematocrit on admission & 0.87 & 0.13 & 0.56 \\
\hline Hematocrit trend during worsening of symptoms & 0.062 & 0.54 & 0.51 \\
\hline CRP on admission & 0.042 & 0.013 & 0.68 \\
\hline CRP during worsening of symptoms & 0.62 & 0.169 & 0.067 \\
\hline D-dimer on admission & 0.11 & 0.003 & 0.003 \\
\hline D-dimer during worsening symptoms & 0.46 & 0.17 & 0.07 \\
\hline LDH on admission & 0.50 & 0.23 & 0.015 \\
\hline LDH during worsening of symptoms & 0.60 & 0.08 & 0.106 \\
\hline Ferritin on admission & 0.13 & 0.317 & 0.28 \\
\hline Ferritin during worsening of symptoms & 0.29 & 0.20 & 0.24 \\
\hline ALT on admission & 0.17 & 0.029 & 0.015 \\
\hline ALT trend during worsening of symptoms & 0.25 & 0.003 & 0.015 \\
\hline
\end{tabular}

\section{TABLE 2: Summary of effects of all variables on three outcomes}

BP, blood pressure; TLC, total leukocyte count; MCV, mean corpuscular volume; CRP, C-reactive protein; LDH, lactate dehydrogenase; ALT, alanine transaminase

Platelet counts were most strongly associated with all study outcomes, followed by mean corpuscular volume (MCV), most associated with mortality, hematocrit with length of hospital stay, and hemoglobin and total leukocyte count (TLC) with severity. Platelet count levels were associated with worsening symptoms and more extended hospital stay $(\mathrm{p}=0.07)$; reduced platelet count was associated with more severe illness and more mortality $(\mathrm{p}=0.016, \mathrm{p}=0.044$, respectively; Table 2$)$.

MCV that increased during a worsening of symptoms affected the patient mortality (Table 2, Table 3) but not the severity and length of stay. Hemoglobin on admission affected the severity of illness: hemoglobin levels within reference range on admission were associated with more severe illness than low hemoglobin levels ( $p$ $=0.05 ;$ Table 2 ). Reduced hematocrit during a worsening of symptoms was associated with prolonged hospital stay with borderline significance $(\mathrm{p}=0.063$; Table 2$)$. 


\section{Cureus}

\begin{tabular}{|c|c|c|c|c|}
\hline \multirow{2}{*}{ Variable } & \multirow{2}{*}{ Trend } & \multicolumn{2}{|l|}{ Mortality } & \multirow{2}{*}{ p-Value } \\
\hline & & Died & Survived & \\
\hline \multirow{3}{*}{ Platelets trend during worsening of symptoms } & Increasing & $1(4.5 \%)$ & $21(95.5 \%)$ & \multirow{3}{*}{0.044} \\
\hline & Decreasing & $3(37.5 \%)$ & $5(62.5 \%)$ & \\
\hline & Static & $2(18.2 \%)$ & $9(81.8 \%)$ & \\
\hline \multirow{3}{*}{ MCV trend during worsening of symptoms } & Increasing & $3(50.0 \%)$ & $3(50.0 \%)$ & \multirow{3}{*}{0.049} \\
\hline & Decreasing & $0(0.0 \%)$ & $2(4.4 \%)$ & \\
\hline & Static & $3(9.1 \%)$ & $30(90.9 \%)$ & \\
\hline \multirow{3}{*}{ CRP trend during worsening of symptoms } & Increasing & $3(12.5 \%)$ & $21(87.5 \%)$ & \multirow{3}{*}{0.067} \\
\hline & Decreasing & $3(42.9 \%)$ & $4(57.1 \%)$ & \\
\hline & Static & $0(0 \%)$ & $10(100 \%)$ & \\
\hline \multirow{4}{*}{ LDH on admission (units/L) } & $<200$ & $0(0 \%)$ & $2(100 \%)$ & \multirow{4}{*}{0.015} \\
\hline & $200-500$ & $1(2.9 \%)$ & $33(97.1 \%)$ & \\
\hline & $500-900$ & $3(27.3 \%)$ & $8(72.7 \%)$ & \\
\hline & $900-1,500$ & $2(50 \%)$ & $2(50 \%)$ & \\
\hline \multirow{4}{*}{ D-dimers on admission $(\mu \mathrm{g} / \mathrm{mL})$} & $200-500$ & $0(0 \%)$ & $17(100 \%)$ & \multirow{4}{*}{0.003} \\
\hline & $500-2,000$ & $1(0 \%)$ & $10(100 \%)$ & \\
\hline & $2,000-5,000$ & $3(8.3 \%)$ & $11(91.7 \%)$ & \\
\hline & $>5,000$ & $2(50 \%)$ & $3(50 \%)$ & \\
\hline \multirow{2}{*}{ D-dimers trend during worsening of symptoms } & Increasing & $6(23.1 \%)$ & $20(76.9 \%)$ & \multirow{2}{*}{0.07} \\
\hline & Static & $0(0 \%)$ & $15(100 \%)$ & \\
\hline \multirow{3}{*}{ ALT on admission (units/L) } & $<40$ & $2(4.9 \%)$ & $39(95.1 \%)$ & \multirow{3}{*}{0.015} \\
\hline & $40-80$ & $1(50 \%)$ & $1(50 \%)$ & \\
\hline & $>80$ & $3(37.5 \%)$ & $5(62.5 \%)$ & \\
\hline \multirow{3}{*}{ ALT trend during worsening of symptoms } & Increasing & $3(30 \%)$ & $7(70 \%)$ & \multirow{3}{*}{0.015} \\
\hline & Decreasing & $1(100 \%)$ & $0(0 \%)$ & \\
\hline & Static & $2(6.7 \%)$ & $28(93.3 \%)$ & \\
\hline
\end{tabular}

\section{TABLE 3: Association of variables affecting mortality $(n=51)$}

MCV, mean corpuscular volume; CRP, C-reactive protein; ALT, alanine transaminase

Lactate dehydrogenase (LDH) on admission affected mortality $(\mathrm{p}=0.012)$, and most patients with LDH above $500 \mathrm{U} / \mathrm{L}$ died (Table 3). While LDH did not affect severity significantly, most patients with LDH ranging from 500 to $900 \mathrm{U} / \mathrm{L}$ had severe illness. C-reactive protein levels during a worsening of symptoms correlated to mortality with borderline significance: when CRP decreased during worsening symptoms, three of seven patients died $(\mathrm{p}=0.067$; Table 3$)$.

D-dimer levels on admission above 2,000 $\mathrm{ng} / \mathrm{mL}$ were associated with severe and critical illness in most cases $(p=0.003)$ and five of six mortalities $(p=0.003)$. During worsening of symptoms, all patients had either increasing or static D-dimer levels, and all six patients who died had increasing D-dimer levels $(\mathrm{p}=$ 0.07). Ferritin on admission and its trends did not affect any study outcomes.

Alanine transaminase (ALT) $>80 \mathrm{mg} / \mathrm{dL}$ on admission was associated with mortality $(\mathrm{p}=0.015)$ and with severe to critical illness $(p=0.029)$. More mortality $(p=0.015)$ and more severity $(p=0.003)$ were associated 
with ALT increasing during the worsening of symptoms. However, one patient had a fall in ALT during a worsening of hypoxia after an initial rise, and he died; this observation needs further examination from other studies.

\section{Discussion}

Despite a rapidly increasing understanding of the pathogenesis of COVID-19, uncertainty remains about clinical symptoms and optimal management [8], so the need for further research is inevitable.

Lippi et al. observed that most published articles have discussed the clinical features and imaging findings of COVID-19 patients, yet few studies have addressed the diagnostic and prognostic value of abnormal laboratory findings [9]. Irrespective of its inherent definition [10], this branch of medical science is effectively involved in epidemiologic surveillance and prognosis determination.

We chose two clinical indices, five common complete blood count (CBC), and five non-CBC blood indices, and retrospectively followed them with the patient's clinical stage of severity and observed their impacts on outcomes. We observed that these indices fluctuated with the course of the illness - some had an impact on outcomes, and some did not. This observation can be used to rationalize laboratory testing.

Zhou et al. conducted a meta-analysis and concluded that comorbidities, including obesity, hypertension, and diabetes, were clinical risk factors for a severe or fatal outcome associated with COVID-19 [11]. Our study observations on age and gender align with the literature, but our findings for comorbidities and treatment did not align with the literature. Racial differences and lack of awareness might cause these observed differences.

For those who survived COVID-19, hypotension during maximum symptoms and oxygen saturation on admission $<80 \%$ led to prolonged hospital stay, and hence correlated to poorer COVID-19 outcomes [12].

Yang et al. conducted a meta-analysis of nine studies and reported a low platelet count associated with an increased risk of severe disease and mortality [13]. Our findings align with Yang's, but we further conclude that reduced platelet count with worsening of the clinical situation was correlated with mortality, and increased platelet count correlated with more severe (non-critical) illness and more extended hospital stay. Therefore, platelet count trends in any direction might predict COVID-19 outcomes.

Most TLC studies reported that leukopenia or normal counts in these patients were associated with poorer outcomes [14]. Because most of our patients had elevated TLC (probably due to over-the-counter steroid use or superimposed infection), we observed more severe illness with rising TLC during a worsening of symptoms.

Lippi et al. concluded that in COVID-19 infection, a progressive decrease in the hemoglobin concentration might reflect a worse clinical progression [15]. Our study results showed that normal hemoglobin on admission, compared with low hemoglobin, was associated with more severe disease. This finding may be more linked to the generally high financial group coming to private hospitals and the high-altitude residence of most study subjects, so relative anemia cannot be excluded. Additional evaluation with metaanalysis from different financial and altitude groups is warranted.

MCV rise with disease progression might be indicative of the development of leukoerythroblastic picture with disease course and, therefore, mortality in our study as Mitra et al. described in their article. However, they were only reporting the findings of a single patient with COVID-19, and they aimed to describe the finding of leukoerythroblastosis in this viral infection [16].

In our study, hospital stay was prolonged with decreasing hematocrit with worsening symptoms, just as Wang et al. concluded that low hematocrit was a predictor of poor outcomes [17].

Sun et al. noted in COVID-19 patients that liver function tests, including aspartate transaminase, ALT, gamma-glutamyl transferase, and LDH, were elevated [18]. Huang associated elevated ALT with more severe illness [4]. Our study results endorse these findings and suggest that ALT is a robust prognostic marker.

Ye et al. concluded that D-dimer's initial and peak values in deceased COVID-19 patients were higher [19]. We found D-dimer as one of the more critical prognostic factors affecting our study outcomes.

Dong et al. reported that LDH levels in COVID-19 patients who died were significantly higher than those who survived [20]. Our study findings endorsed Dong's findings, as we found LDH on admission and its trend as a useful prognostic marker.

While Cheng et al. suggested that ferritin was associated with poor prognosis in COVID-19 patients [21], Feld et al. reported that elevated ferritin levels were not accurate predictors of outcomes [22]. Our study 
results align with Feld's finding, indicating against testing ferritin levels repeatedly as a prognostic marker of COVID-19 disease.

Chen et al. demonstrated that the plasma CRP level was positively correlated to the severity of COVID-19 [23]. In our study, elevated CRP on admission was associated with more severe disease and extended hospital stay. During worsening of symptoms, a reduction in CRP was significantly correlated with more mortality, likely indicating a decline in the patient's immune response.

Our small sample size limited our study, and we recommend larger trials to confirm our findings. We did not correlate our findings with disease complications due to missing data, so future studies should compensate for this accordingly.

\section{Conclusions}

We evaluated the association between laboratory investigations and their effects on COVID-19 outcomes to determine if multiple laboratory evaluations are clinically justified. Patient age, gender, comorbid conditions, drugs, residence, and ferritin levels did not affect disease outcomes. Hemoglobin, platelet count, MCV, CRP, D-dimer, ALT, LDH, hypoxia, and hypotension were all correlated to disease outcomes.

Therefore, these factors are sufficient, justifiable laboratory examinations for COVID-19 patients, especially in poor countries where resources and funds for laboratory examinations are limited.

\section{Additional Information}

\section{Disclosures}

Human subjects: Consent was obtained or waived by all participants in this study. Institutional Review Board And Ethics Committee, Shifa International Hospital Islamabad issued approval 121-941-2020. "Covid Clinical and Laboratory Parameters: Dismembering the Values, Reveals Outcome; An Observational Cohort Study" has been reviewed by Institutional Review Board And Ethics Committee of Shifa International Hospital Islamabad. IRB \& Ethics Committee is pleased to approve the study. Animal subjects: All authors have confirmed that this study did not involve animal subjects or tissue. Conflicts of interest: In compliance with the ICMJE uniform disclosure form, all authors declare the following: Payment/services info: All authors have declared that no financial support was received from any organization for the submitted work. Financial relationships: All authors have declared that they have no financial relationships at present or within the previous three years with any organizations that might have an interest in the submitted work. Other relationships: All authors have declared that there are no other relationships or activities that could appear to have influenced the submitted work.

\section{References}

1. Adhikari SP, Meng S, Wu YJ, et al.: Epidemiology, causes, clinical manifestation and diagnosis, prevention and control of coronavirus disease (COVID-19) during the early outbreak period: a scoping review. Infect Dis Poverty. 2020, 9:29. 10.1186/s40249-020-00646-x

2. World Health Organization: Statement on the Second Meeting of the International Health Regulations (2005) Emergency Committee Regarding the Outbreak of Novel Coronavirus (2019-nCoV). (2020). Accessed: February 2, 2021: https://www.who.int/news-room/detail/30-01-2020-statement-on-the-second-meetingof-the-international-health-regulatio...

3. Hu B, Ge X, Wang LF, Shi Z: Bat origin of human coronaviruses. Virol J. 2015, 12:221. 10.1186/s12985-0150422-1

4. Huang C, Wang Y, Li X, et al.: Clinical features of patients infected with 2019 novel coronavirus in Wuhan, China. Lancet. 2020, 395:497-506. 10.1016/S0140-6736(20)30183-5

5. Wang D, Hu B, Hu C, et al.: Clinical characteristics of 138 hospitalized patients with 2019 novel coronavirus-infected pneumonia in Wuhan, China. JAMA. 2020, 323:1061-1069. 10.1001/jama.2020.1585

6. Chen N, Zhou M, Dong X, et al.: Epidemiological and clinical characteristics of 99 cases of 2019 novel coronavirus pneumonia in Wuhan, China: a descriptive study. Lancet. 2020, 395:507-513. 10.1016/S01406736(20)30211-7

7. Singh AK, Singh A, Shaikh A, Singh R, Misra A: Chloroquine and hydroxychloroquine in the treatment of COVID-19 with or without diabetes: a systematic search and a narrative review with a special reference to India and other developing countries. Diabetes Metab Syndr. 2020, 14:241-246. 10.1016/j.dsx.2020.03.011

8. Wiersinga WJ, Rhodes A, Cheng AC, Peacock SJ, Prescott HC: Pathophysiology, transmission, diagnosis, and treatment of coronavirus disease 2019 (COVID- 19): a review. JAMA. 2020, 324:782-793. 10.1001/jama.2020.12839

9. Lippi G, Plebani M: Laboratory abnormalities in patients with COVID-2019 infection. Clin Chem Lab Med. 2020, 58:1131-1134. 10.1515/cclm-2020-0198

10. Lippi G, Plebani M: A modern and pragmatic definition of laboratory medicine . Clin Chem Lab Med. 2020, 58:1171. 10.1515/cclm-2020-0114

11. Zhou Y, Yang Q, Chi J, Dong B, Lv W, Shen L, Wang Y: Comorbidities and the risk of severe or fatal outcomes associated with coronavirus disease 2019: a systematic review and meta-analysis. Int J Infect Dis. 2020, 99:47-56. 10.1016/j.ijid.2020.07.029

12. Bhatraju PK, Ghassemieh BJ, Nichols M, et al.: Covid-19 in critically ill patients in the Seattle region - case series. N Engl J Med. 2020, 382:2012-2022. 10.1056/NEJMoa2004500

13. Yang AP, Liu JP, Tao WQ, Li HM: The diagnostic and predictive role of NLR, d-NLR and PLR in COVID-19 


\section{Cureus}

patients. Int Immunopharmacol. 2020, 84:106504. 10.1016/j.intimp.2020.106504

14. Lippi G, Plebani M, Henry BM: Thrombocytopenia is associated with severe coronavirus disease 2019 (COVID-19) infections: a meta-analysis. Clin Chim Acta. 2020, 506:145-148. 10.1016/j.cca.2020.03.022

15. Lippi G, Mattiuzzi C: Hemoglobin value may be decreased in patients with severe coronavirus disease 2019 . Hematol Transfus Cell Ther. 2020, 42:116-117. 10.1016/j.htct.2020.03.001

16. Mitra A, Dwyre DM, Schivo M, Thompson 3rd GR, Cohen SH, Ku N, Graff JP: Leukoerythroblastic reaction in a patient with COVID-19 infection. Am J Hematol. 2020, 95:999-1000. 10.1002/ajh.25793

17. Wang C, Zhang H, Cao X, et al.: Red cell distribution width (RDW): a prognostic indicator of severe COVID19. Ann Transl Med. 2020, 8:1230. 10.21037/atm-20-6090

18. Sun Y, Dong Y, Wang L, Xie H, Li B, Chang C, Wang F-S: Characteristics and prognostic factors of disease severity in patients with COVID- 19: the Beijing experience. J Autoimmun. 2020, 112:102473. 10.1016/j.jaut.2020.102473

19. Ye W, Chen G, Li X, et al.: Dynamic changes of D-dimer and neutrophil-lymphocyte count ratio as prognostic biomarkers in COVID-19. Respir Res. 2020, 21:169. 10.1186/s12931-020-01428-7

20. Dong X, Sun L, Li Y: Prognostic value of lactate dehydrogenase for in-hospital mortality in severe and critically ill patients with COVID-19. Int J Med Sci. 2020, 17:2225-2231. 10.7150/ijms.47604

21. Cheng L, Li H, Li L, Liu C, Yan S, Chen H, Li Y: Ferritin in the coronavirus disease 2019 (COVID- 19): a systematic review and meta-analysis. J Clin Lab Anal. 2020, 34:e23618. 10.1002/jcla.23618

22. Feld J, Tremblay D, Thibaud S, Kessler A, Naymagon L: Ferritin levels in patients with COVID- 19: a poor predictor of mortality and hemophagocytic lymphohistiocytosis. Int J Lab Hematol. 2020, 42:773-779. 10.1111/ijlh.13309

23. Chen W, Zheng KI, Liu S, Yan Z, Xu C, Qiao Z: Plasma CRP level is positively associated with the severity of COVID-19. Ann Clin Microbiol Antimicrob. 2020, 19:18. 10.1186/s12941-020-00362-2 